PHYSICAL REVIEW D 90, 107502 (2014)

\title{
Estimates of maximum energy density of cosmological gravitational-wave backgrounds
}

\author{
John T. Giblin, Jr. ${ }^{1,2, *}$ and Eric Thrane ${ }^{3, \dagger}$ \\ ${ }^{1}$ Department of Physics, Kenyon College, Gambier, Ohio 43022, USA \\ ${ }^{2}$ Department of Physics, Case Western Reserve University, Cleveland, Ohio 44106, USA \\ ${ }^{3}$ LIGO Laboratory, California Institute of Technology, MS 100-36, Pasadena, California 91125, USA
}

(Received 20 June 2014; published 24 November 2014)

\begin{abstract}
The recent claim by BICEP2 of evidence for primordial gravitational waves has focused interest on the potential for early-Universe cosmology using gravitational waves. In addition to cosmic microwave background detectors, efforts are underway to carry out gravitational-wave astronomy with pulsar timing arrays, space-based detectors, and terrestrial detectors. These efforts will probe a wide range of times in the early Universe, during which backgrounds may have been produced through processes such as phase transitions or preheating. We derive a rule of thumb (not so strong as an upper limit) governing the maximum energy density of cosmological backgrounds. For most scenarios, we expect the energy density spectrum to peak at values of $\Omega_{\mathrm{gw}}(f) \lesssim 10^{-12 \pm 2}$. We discuss the applicability of this rule and the implications for gravitational-wave astronomy.
\end{abstract}

DOI: $10.1103 /$ PhysRevD.90.107502

PACS numbers: 04.30.Db, 04.30.-w, 04.80.Nn, 98.80.Es

Pending confirmation, the detection by BICEP2 of primordial gravitational waves (GWs) has launched the era of GW astronomy [1]. Cosmic microwave background detectors observe GWs at low frequencies $f \lesssim 10^{-16} \mathrm{~Hz}$. The direct detection of GWs by other observatories at higher frequencies is likely imminent. In the coming years, a network of terrestrial GW detectors [2-5], operating at $\sim 10-2000 \mathrm{~Hz}$, is expected to detect dozens of compact binary coalescences per year [6]. Pulsar timing arrays, operating at $\sim 5 \mathrm{nHz}$, are approaching the sensitivity required for the GW detection from supermassive black hole binaries [7]. Space-based detectors [8], operating at $0.1 \mathrm{mHz}-1 \mathrm{~Hz}$, are all but guaranteed to observe GWs from, e.g., merging supermassive black holes.

Cosmological backgrounds, created by major events in the history of the Universe, can be created from or following inflation [9-11], by phase transitions [12,13], and from alternative cosmologies [14]. Astrophysical backgrounds are also expected to arise from the superposition of many unresolvable signals [15-19]. While astrophysical backgrounds are interesting in their own right, we focus here on cosmological backgrounds.

Recent observations by BICEP2 [1] of a primordial background are most simply explained as the amplification of vacuum fluctuations following inflation [20], implying a background with a nearly flat energy density spectrum:

$$
\Omega_{\mathrm{gw}}(f) \equiv \frac{1}{\rho_{c}} \frac{d \rho_{\mathrm{gw}}}{d \ln f}
$$

Here, $\rho_{\mathrm{gw}}$ is GW energy density, $f$ is frequency, and $\rho_{c}$ is the critical energy density required for a closed Universe.

*giblinj@kenyon.edu

ethrane@ligo.caltech.edu
Extrapolating the BICEP2 measurement to the LIGO band, we expect $\Omega_{\mathrm{gw}}(f) \approx 10^{-15}$. GW backgrounds at this level are too weak to observe directly except by the most ambitious detectors [8] and, of course, using the cosmic microwave background [1].

However, cosmological signals, produced through other mechanisms, can produce considerably more detectable signals with $\Omega_{\mathrm{gw}} \lesssim 10^{-12}$. Detection of a cosmological background above the level predicted for the amplification of vacuum fluctuations could point to a richer and more interesting early Universe than posited by the simplest version of slow-roll inflation.

Here, we draw attention to generic features common to many cosmological backgrounds in order to derive a "rule of thumb" governing the maximum likely amplitude of most cosmological backgrounds. We use the phrase rule of thumb rather than upper limit to convey the theoretical uncertainty in our derivation. The rule of thumb employs assumptions consistent with a large number of models in order to provide a broadly (if not universally) applicable prediction governing the maximum energy density of cosmological backgrounds. The point is to provide a systematic framework for understanding trends among predictions of cosmological backgrounds.

The rule applies to backgrounds (at all energy scales) created after inflation during the radiation-dominated epoch. During this epoch, the age of the Universe varied between $10^{-35} \mathrm{~s} \lesssim t \lesssim 47000 \mathrm{yr}$, corresponding to energy scales of $10^{15} \mathrm{GeV} \gtrsim E \gtrsim 1 \mathrm{eV}$, and redshifts $z \gtrsim 3500$ [21]. We assume that the length scale of the source is smaller than the cosmological horizon $\mathrm{H}^{-1}$, which follows from causality. The rule does not apply to astrophysical backgrounds, which can peak above cosmological models [16], as they are created at much later times. 
We assume that the background evolves as a FriedmannLemäitre-Robertson-Walker spacetime. GWs propagate as strain perturbations $h_{i j}$ in synchronous gauge, where the propagating degrees of freedom are the two polarizations that obey the transverse-traceless conditions and the sourced Klein-Gordon equations,

$$
\ddot{h}_{i j}+3 H \dot{h}_{i j}-\frac{1}{a^{2}} \nabla^{2} h_{i j}=(16 \pi G) S_{i j}^{T T} .
$$

The source is the transverse-traceless projection of the anisotropic stress tensor: $S_{i j}^{T T}=T_{i j}-\left(\delta_{i j} / 3\right) T_{k}^{k}$.

Our objective is to estimate $\Omega_{\mathrm{gw}}(f)$ from a relatively generic cosmological source. To this end, we link GW energy density $\rho_{\mathrm{gw}}$ to the energy density of some source $\rho_{\mathrm{gw}}<\rho_{s}$, which, in turn, represents some fraction of the total energy density in the Universe $\rho_{s}<\rho$. By considering the fraction of energy density available for the source, and the fraction of the source energy density converted to GWs, we estimate the maximum $\Omega_{\mathrm{gw}}(f)$ today.

We make a few assumptions about the source. We consider a source associated with a characteristic scale $k_{*}$, and assume that components of the stress-energy tensor can be written in momentum space as

$$
\tilde{T}_{i j}(\vec{k}) \approx \tilde{T}(\vec{k})=A \exp \left[-\frac{\left(|\vec{k}|-k_{*}\right)^{2}}{2 \sigma^{2}}\right],
$$

where each $T_{i j}(k)$ is approximately the same magnitude, $\sigma$ parametrizes the source width, and $A$ is the peak height. Although $A$ is determined by the detailed physics of each source, it cannot exceed the total energy density of the Universe at the time of the process.

The isotropic pressure of the source $\tilde{p}_{s}(\vec{k})=$ $\operatorname{Tr}\left[\tilde{T}_{i j}(\vec{k})\right] / 3=\tilde{T}(\vec{k})$, is related to energy density:

$$
\tilde{\rho}_{s}(\vec{k})=\frac{\tilde{p}_{s}(\vec{k})}{w}=\frac{\tilde{T}(\vec{k})}{w},
$$

by $w$, which relates the magnitude of the stress-energy tensor of the source to the source energy density. If we chose a volume large enough so the configuration-space energy density is homogeneous, we can use Parseval's theorem to relate the momentum space energy spectrum to the total source energy in a volume $V$,

$$
\int d^{3} k\left|\tilde{\rho}_{s}(\vec{k})\right|^{2}=\int d V \rho_{s}^{2}(\vec{x}) \approx V \rho_{s}^{2} .
$$

We define

$$
W\left(k_{*}, \sigma\right) \equiv 4 \pi \int_{0}^{\infty} k^{2} \exp \left[-\frac{\left(k-k_{*}\right)^{2}}{\sigma^{2}}\right] d k
$$

The magnitude of $\tilde{T}$ and the source energy density are related: $|A|^{2}=w^{2} \rho_{s}^{2} V / W\left(k_{*}, \sigma\right)$. The GW energy created in this process is only a fraction, $\alpha<1$ of the energy budget of the Universe: $\rho_{s}=\alpha \rho$. Thus,

$$
|\tilde{T}|^{2}=\frac{w^{2} \alpha^{2} V \rho^{2}}{W\left(k_{*}, \sigma\right)} \exp \left[-\frac{\left(k-k_{*}\right)^{2}}{\sigma^{2}}\right] .
$$

Next, we calculate the size of the metric perturbations. Since each mode $h_{i j}(\vec{k})$ obeys Eq. (2) (assuming that the source is short lived compared to the Hubble time, allowing us to momentarily ignore the Hubble Friction term), we estimate the maximum size of $\tilde{h}_{i j}$ by studying the point when the acceleration of $h_{i j}(\vec{k})$ vanishes. In the language of a harmonic oscillator, we balance the force due to the source with the restoring force. It follows that the $h_{i j}$ are approximately the same:

$$
\tilde{h} \approx \tilde{h}_{i j} \approx \frac{16 \pi G}{k^{2}} S^{T T} .
$$

Last, we define: $\beta \equiv\left|S^{T T}\right|^{2} /|T|^{2}$. The projection of $T_{i j}$ onto $S_{i j}^{T T}$ extracts the tensor part of the stress-energy tensor and is therefore sensitive to the source geometry. This is the hardest parameter to estimate without specific knowledge of the source.

We determine the magnitude of $A(\vec{k})$, but not the phase, necessary to estimating $\beta$. One realization of $A_{i j}=|A| e^{i \theta_{i j}}$ has six independent phases. We randomly chose six phases and project the stress-energy tensor $T_{i j}$ onto the transverse-traceless anisotropic stress tensor $S_{i j}^{T T}$ generating a distribution of $\beta$. Using a simulation, we determine $\bar{\beta} \approx$ $10^{-1.5}-10^{-2}$ for a random process.

We use

$$
\Omega_{\mathrm{gw}}(k)=\frac{1}{\rho} \frac{k^{3}}{32 \pi G} \frac{1}{V} \sum_{i, j} \int d \Omega\left|\dot{h}_{i j}^{\mathrm{TT}}(t, \mathbf{k})\right|^{2}
$$

to calculate $\Omega_{\mathrm{gw}}(k)$ at the time when the source vanishes [22]. We exchange numerical factors for the sum in Eq. (9) and evaluate the angular part of the integral,

$$
\begin{gathered}
\sum_{i j} \int d \Omega\left|\dot{\tilde{h}}_{i j}\right|^{2}=36 \pi|\dot{\tilde{h}}|^{2} \\
|\dot{\tilde{h}}|^{2}=|\tilde{h}|^{2} k^{2}=(16 \pi G)^{2} \frac{\beta|\tilde{T}|^{2}}{k^{2}}
\end{gathered}
$$

via Eq. (8). We combine Eq. (11) with (10) and plug into Eq. (9), yielding

$$
\Omega_{\mathrm{gw}}(k)=\frac{288 \pi^{2} G}{\rho V} k \beta|\tilde{T}|^{2}=\frac{108 \pi}{\rho^{2} V} H^{2} k \beta^{2}|\tilde{T}|^{2},
$$

where the final equality follows from Friedmann's equation: $H^{2}=(8 \pi G / 3) \rho$. When the source vanishes, 


$$
\Omega_{\mathrm{gw}}(k)=108 \pi \alpha^{2} \beta w^{2} \frac{H^{2} k}{W\left(k_{*}, \sigma\right)} e^{\left[-\left(k-k_{*}\right)^{2} / \sigma^{2}\right]} .
$$

It might seem surprising that we can write this spectrum so simply. In particular, Eq. (13) depends on the dimensionless quantity $H^{2} k / W\left(k_{*}, \sigma\right)$, and so we need not know the scale $k_{*}$. The peak energy density can be estimated by evaluating $\Omega_{\mathrm{gw}}\left(k_{*}\right) \approx 108 \pi \alpha^{2} \beta w^{2} N\left(k_{*}, \sigma\right)$.

Last,

$$
N\left(k_{*}, \sigma\right) \equiv \frac{H^{2} k_{*}}{W\left(k_{*}, \sigma\right)}=\frac{\left(k_{*} H^{-1}\right)}{W\left(k_{*} H^{-1}, \sigma H^{-1}\right)} .
$$

We study Eq. (14) numerically. For fixed $k_{*}, N\left(k_{*}, \sigma\right)$ diverges as $\sigma \rightarrow 0$, but only for unphysically small $\sigma$. Generally, the source width can be a few orders of magnitude smaller than the characteristic frequency. Nonetheless, decreasing the source width changes the amplitude of the GW spectrum modestly. For small values of $\sigma / k_{*}, N\left(k_{*}, \sigma\right) \propto k_{*} / \sigma$.

In practice, we expect $\sigma<k_{*}$, so we estimate $N\left(k_{*}, \sigma\right)$ by setting the ratio of $\sigma / k_{*}$. In the small $\sigma / k_{*}$ limit,

$$
N\left(k_{*}, \sigma\right) \rightarrow 0.045\left(\frac{k_{*}}{\sigma}\right)\left(\frac{H}{k_{*}}\right)^{2},
$$

where 0.045 is obtained evaluating $W\left(k_{*}, \sigma\right)$ numerically. In present times [22,25],

$$
\Omega_{\mathrm{gw}, 0}(k) h^{2}=\Omega_{\mathrm{rad}, 0} h^{2}\left(g_{0} / g_{e}\right)^{1 / 3} \Omega_{\mathrm{gw}}(k)
$$

where 0 indicates the present, $h$ is the dimensionless Hubble parameter, and $\Omega_{\text {rad }, 0}$ is the current energy density from radiation. The factor $g_{0} / g_{\mathrm{e}} \approx 1 / 10$ is the ratio of the number of degrees of freedom today to the number at matter-radiation equality. We adopt $h=0.68$ [21].

Since $\Omega_{\mathrm{rad}, 0}=7.78 \times 10^{-5}$ [21], we obtain

$$
\Omega_{\mathrm{gw}, 0} \approx 0.012 \alpha^{2} \beta w^{2} N\left(k_{*}, \sigma\right) \frac{k}{k_{*}} e^{\left[-\left(k-k_{*}\right)^{2} / \sigma^{2}\right]} .
$$

The frequency today is related to the wave vector at the time of GW production [22-24]: $f=6 \times 10^{10} \mathrm{~Hz}\left(k / \sqrt{m_{\mathrm{pl}} H}\right)$. The present-day GW frequency is a function only of the energy scale at the time of the source $H$ and the dimensionless constant $k / H$.

There are three tunable parameters: $(\alpha, \beta, w)$. For strong signals $\alpha \lesssim 1$, but $\alpha \approx 0.5$ is more likely. The second parameter, $w \lesssim 1$, is likely. For scalar fields, e.g., $w \approx 1 / 3$. The third parameter $\beta$, describing how inherently quadrupolar the energy density is, has the greatest dynamic range. One can imagine situations in which almost the entire Universe is the source as well as cases in which the source is a small fraction of the energy budget.

Putting everything together, the peak height is

$$
\Omega_{\mathrm{gw}, 0}\left(k_{*}\right) \approx 2.3 \times 10^{-4} \alpha^{2} \beta w^{2} \frac{k_{*}}{\sigma}\left(\frac{H}{k_{*}}\right)^{2} .
$$

(This scaling is noted in [26-28].) We identify plausible values of $k_{*} / H$ and $\sigma / k_{*}$. In principle, $k_{*} / H$ is different for different cosmological processes. However, given our goal of constraining the maximum allowable $\Omega_{\mathrm{gw}}$, we chose a value as small as possible so that $N\left(k_{*}, \sigma\right)$ is as large as possible, subject to constraints from causality: the peak wavelength must be subhorizon. Motivated by models of bubble collisions [29,30] and phase transitions [12,13,31], we chose fiducial values $k_{*}=100 H_{*}$ and $\sigma / k_{*}=1 / 2$. (While a large class of models employs comparable parameters, other choices can be made for specific models-e.g., [26,32] — which can be investigated with Eq. (18).) We thereby obtain our rule of thumb:

$$
\Omega_{\mathrm{gw}, 0}\left(k_{*}\right) \approx 4.7 \times 10^{-8} \alpha^{2} \beta w^{2} .
$$

If we repeat the above calculations assuming that $\tilde{T}(\vec{k})$ is described, not by a Gaussian distribution as in Eq. (3), but by a plateau distribution [constant on $\left(k_{*}-\sigma, k_{*}+\sigma\right)$ and zero everywhere else], then the resulting rule of thumb prediction is just $9 \%$ less. Thus, the results do not depend strongly on the shape of $\tilde{T}(\vec{k})$.

We consider three scenarios-corresponding to three sets of tunable parameters $(\alpha, \beta, w)$-reflecting the plausible range of $\Omega_{\mathrm{gw}}\left(k_{*}\right)$. In Table I these scenarios are labeled "optimistic," "realistic," and "pessimistic." These categorizations, inspired by [6], are necessarily subjective. However, by providing a range of values, we show a range of possible outcomes. For the realistic scenario, the rule of thumb becomes $\Omega_{\mathrm{gw}, 0}\left(k_{*}\right) \approx 1 \times 10^{-12}$.

We consider (i) Advanced LIGO using 1 yr of coincident Hanford-Livingston data at design sensitivity; (ii) the Einstein Telescope using $1 \mathrm{yr}$ of data with the "ET-D" sensitivity [33]; (iii) a hypothetical pulsar timing array from [34] consisting of 20 pulsars assuming $100 \mathrm{~ns}$ timing noise, $5 \mathrm{yr}$ of observation time, and a cadence of $20 \mathrm{yr}^{-1}$; and (iv) the Big Bang Observer (BBO) [35,36], a proposed space-based detector using parameters from [34].

For each detector, we optimistically tune the peak frequency $f_{*} \equiv c k_{*} / 2 \pi$ to produce the most favorable signal. The results are summarized in Fig. 1. The rule-ofthumb signals (thin dashed) are compared to the sensitivity curve for each detector (solid). The sensitivity curves are "power-law integrated curves" [34], representing the sensitivity of each detector to a broadband stochastic background

TABLE I. Energy density peak heights for three sets of tunable parameters assuming $\sigma / k_{*}=1 / 2$ and $k_{*} / H=100$.

\begin{tabular}{llllc}
\hline \hline Scenario & \multicolumn{1}{c}{$\alpha$} & \multicolumn{1}{c}{$\beta$} & \multicolumn{1}{c}{$w$} & $\Omega_{\mathrm{gw}}\left(k_{*}\right)$ \\
\hline Optimistic & 1 & 0.1 & $1 / 3$ & $4.97 \times 10^{-10}$ \\
Realistic & 0.1 & 0.03 & $1 / 3$ & $1.49 \times 10^{-12}$ \\
Pessimistic & 0.02 & 0.005 & $1 / 3$ & $9.93 \times 10^{-15}$ \\
\hline \hline
\end{tabular}




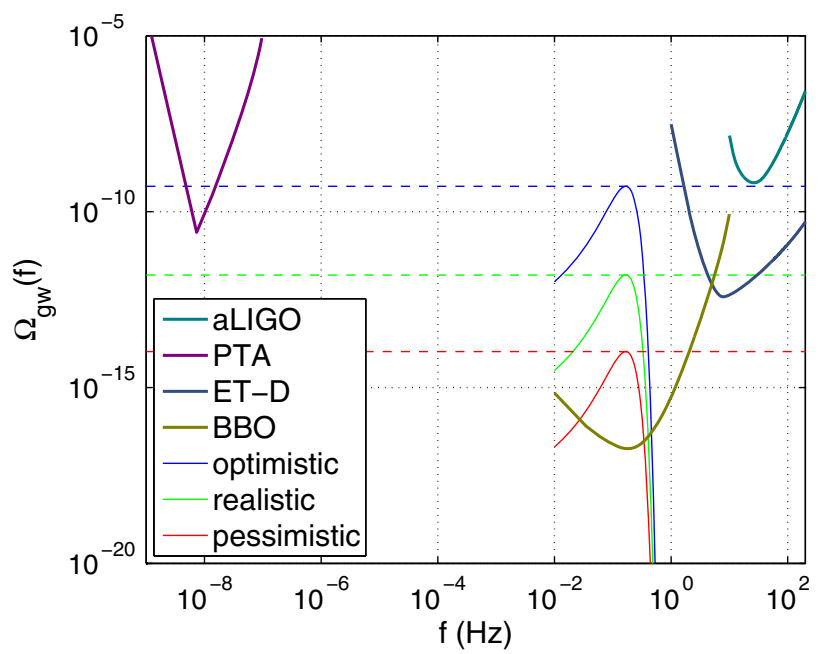

FIG. 1 (color online). Rule-of-thumb energy density spectra compared with sensitivity curves. The thick lines are the powerlaw integrated sensitivity curves [34] for different detectors. The thin dashed lines indicate the peak height for our rule-of-thumb models. To avoid clutter, we plot the rule-of-thumb spectra only for the BBO band. Any signal spectrum falling entirely below the solid power-law integrated curve will produce a signalto-noise ratio of $<1$, and is therefore undetectable. We ignore the difficulties of astrophysical foregrounds and assume that they have been successfully subtracted. We assume a Hubble parameter of $h=0.68$ [21].

with a power-law shape. Any dashed rule-of-thumb line falling below the solid power-law integrated curve is undetectable. Dashed lines intersecting the solid powerlaw integrated curve might be detectable, and when this happens, we calculate the signal-to-noise ratio (SNR) of a two-detector, cross-correlation search [37].

From Fig. 1(a), all three rule-of-thumb spectra are out of reach for Advanced LIGO. The optimistic spectrum can perhaps be probed with additional detectors and/or multiple years of coincident data. The Einstein Telescope detects a highly significant signal from the optimistic spectrum while the realistic spectrum produces a marginal $\mathrm{SNR}=3.2$ detection. The pessimistic spectrum is out of reach. Our hypothetical pulsar timing array unambiguously detects the optimistic spectrum $(\mathrm{SNR}=19)$, but not the realistic or pessimistic spectra. BBO detects statistically significant signatures from all three; SNR $>380$. Note: we have ignored complications arising from correlated noise [38] and the subtraction of astrophysical foregrounds, which may complicate detection.

Our rule of thumb applies to a large subset of cosmological GW sources that occur after inflation and during the radiation-dominated epoch. The argument presented here, after all, relies solely on the ratio of three energy density scales, $\rho_{\mathrm{gw}}<\rho_{s}<\rho$, and on the application of transfer functions. There are, however, exceptions.

In nonminimal inflation $[11,39,40]$, signals "frozen in" during phase transitions evade the bound. GWs remain nondynamical until they re-enter the horizon when the Universe cools to the appropriate temperature, and so the ratio of energy density scales is irrelevant. Another possible modification to inflation involves the introduction of direct couplings between the inflaton (usually an axion) and gauge fields [41-43]. As inflation ends, one polarization of the gauge field is enhanced via a tachyonic process and inflation ends earlier than in canonical inflation. These modes efficiently decay into GWs.

A nonstandard equation of state following inflation might lead to a detectable cosmological background. In particular, a "stiff" equation of state $w>1 / 3$ modifies the expansion history of the Universe, allowing inflationary gravitational radiation to re-enter the horizon with large amplitudes [44]. This model evades our rule of thumb since the source is not postinflationary. However, there is no theoretical motivation for an effective equation of state larger than $1 / 3$ after inflation [44].

If the graviton is not a massless, helicity-2 particle $[45,46]$ this analysis needs to be rethought in the presence of extra degrees of freedom. It is likely that the GW background would be less diluted when the source is projected, leading potentially to $\beta \approx 1$. Thus, it may be easier for observatories to detect cosmological backgrounds in nonstandard theories of gravity [47].

Cosmic string networks produced during phase transitions in the early Universe [48] can produce GWs in the late Universe [19]. The peak wavelength is tied to the size of the cosmic strings, not the Hubble scale, and the background is produced at fairly late times (even though the strings themselves are formed very early). If cosmic string networks were to radiate gravitationally during the radiationdominated era, these signals would be subject to the constraints presented here, where $k_{*} \gg H$ (since strings are small compared to the Hubble scale) likely corresponding to very weak signals today.

Our knowledge of the early Universe is far from precise, and GW astronomy affords us the chance to learn more about this important era. As we prepare for the upcoming era of GW cosmology, it is useful to consider our expectations for what we might reasonably detect based on our present knowledge. To this end, we have proposed a rule of thumb governing the maximum amplitude of GW backgrounds: we expect cosmological backgrounds to produce energy density spectra that peak around $\Omega_{\mathrm{gw}} \approx 10^{-12}$. Our rule of thumb is based on simple scaling arguments and provides robust, if approximate, theoretical guidance for GW cosmology.

In order to evade the rule-of-thumb assumptions, models typically employ assumptions about inflationary dynamics that require some degree of fine-tuning. We argue that, based on our current understanding of the early Universe, the simplest, most natural models predict cosmological GW backgrounds that follow the rule of thumb. We note that observational constraints from big bang nucleosynthesis and 
the cosmic microwave background limit the integrated energy density of cosmological backgrounds; see, e.g., [49].

\section{ACKNOWLEDGMENTS}

J. T. G. is supported by the National Science Foundation, Grant No. PHY-1068080. E. T. is a member of the LIGO
Laboratory, supported by funding from the United States National Science Foundation. LIGO was constructed by the California Institute of Technology and Massachusetts Institute of Technology with funding from the National Science Foundation and operates under cooperative agreement Grant No. PHY-0757058.
[1] P. A. R. Ade et al., Phys. Rev. Lett. 112, 241101 (2014).

[2] G. M. Harry (LIGO Scientific Collaboration), Classical Quantum Gravity 27, 084006 (2010).

[3] F. Acernese (Virgo Collaboration), Classical Quantum Gravity 23, S63 (2006).

[4] K. Somiya (KAGRA Collaboration), Classical Quantum Gravity 29, 124007 (2012).

[5] H. Grote (LIGO Scientific Collaboration), Classical Quantum Gravity 25, 114043 (2008).

[6] J. Abadie et al., Classical Quantum Gravity 27, 173001 (2010).

[7] R. M. Shannon et al., Science 342, 334 (2013).

[8] C. Ungarelli, P. Corasaniti, R. A. Mercer, and A. Vecchio, Classical Quantum Gravity 22, S955 (2005).

[9] L. P. Grishchuk, Sov. Phys. JETP 40, 409 (1975).

[10] A. A. Starobinskii, JETP Lett. 30, 682 (1979).

[11] N. Barnaby, E. Pajer, and M. Peloso, Phys. Rev. D 85, 023525 (2012).

[12] A. Kosowsky, M. S. Turner, and R. Watkins, Phys. Rev. Lett. 69, 2026 (1992).

[13] M. Kamionkowski, A. Kosowsky, and M. S. Turner, Phys. Rev. D 49, 2837 (1994).

[14] V. Mandic and A. Buonanno, Phys. Rev. D 73, 063008 (2006).

[15] A. H. Jaffe and D. C. Backer, Astrophys. J. 583, 616 (2003).

[16] C. Wu, V. Mandic, and T. Regimbau, Phys. Rev. D 85, 104024 (2012).

[17] A. J. Farmer and E. Phinney, Mon. Not. R. Astron. Soc. 346, 1197 (2003).

[18] T. Regimbau and J.A. de Freitas Pacheco, Astron. Astrophys. 376, 381 (2001).

[19] V. Berezinsky, B. Hnatyk, and A. Vilenkin, Baltic Astron. 13, 289 (2004).

[20] A. H. Guth, Phys. Rev. D 23, 347 (1981).

[21] P. Ade et al. (Planck Collaboration), Astron. Astrophys. 66, A16 (2014).

[22] R. Easther, J. T. Giblin Jr., and E. A. Lim, Phys. Rev. Lett. 99, 221301 (2007).

[23] R. Easther and E. A. Lim, J. Cosmol. Astropart. Phys. 04 (2006) 010.
[24] M. A. Amin , M. P. Hertzberg. D. I. Kaiser, and J. Karouby, arXiv:1410.3808.

[25] L. R. Price and X. Siemens, Phys. Rev. D 78, 063541 (2008).

[26] P. Binétruy, A. Bohé, C. Caprini, and J.-F. Dufaux, J. Cosmol. Astropart. Phys. 06 (2012) 027.

[27] J. F. Dufaux, Phys. Rev. Lett. 103, 041301 (2009).

[28] C. Caprini, R. Durrer, and G. Servant, Phys. Rev. D 77, 124015 (2008).

[29] A. Kosowsky, M. S. Turner, and R. Watkins, Phys. Rev. D 45, 4514 (1992).

[30] A. Kosowsky and M. S. Turner, Phys. Rev. D 47, 4372 (1993).

[31] C. Caprini and R. Durrer, Phys. Rev. D 74, 063521 (2006).

[32] S. J. Huber and T. Konstandin, J. Cosmol. Astropart. Phys. 05 (2008) 017.

[33] Einstein Telescope, http://www.et-gw.eu/etsensitivities.

[34] E. Thrane and J. D. Romano, Phys. Rev. D 88, 124032 (2013).

[35] S. Phinney et al., NASA Mission Concept Study (2004).

[36] C. Cutler and J. Harms, Phys. Rev. D 73, 042001 (2006).

[37] B. Allen and J. D. Romano, Phys. Rev. D 59, 102001 (1999).

[38] E. Thrane, N. Christensen, and R. M. S. Schofield, Phys. Rev. D 87, 123009 (2013).

[39] A. Lopez and K. Freese, arXiv:1305.5855.

[40] N. Barnaby and Z. Huang, Phys. Rev. D 80, 126018 (2009).

[41] T. Prokopec, arXiv:astro-ph/0106247.

[42] M. M. Anber and L. Sorbo, Phys. Rev. D 81, 043534 (2010).

[43] N. Barnaby, R. Namba, and M. Peloso, J. Cosmol. Astropart. Phys. 04 (2011) 009.

[44] L. A. Boyle and A. Buonanno, Phys. Rev. D 78, 043531 (2008).

[45] C. de Rham, G. Gabadadze, and A. J. Tolley, Phys. Lett. B 711, 190 (2012).

[46] C. de Rham, Living Rev. Relativity 17, 7 (2014).

[47] S. J. Chamberlin and X. Siemens, Phys. Rev. D 85, 082001 (2012).

[48] T. Kibble, J. Phys. A 9, 1387 (1976).

[49] I. Sendra and T. L. Smith, Phys. Rev. D 85, 123002 (2012). 